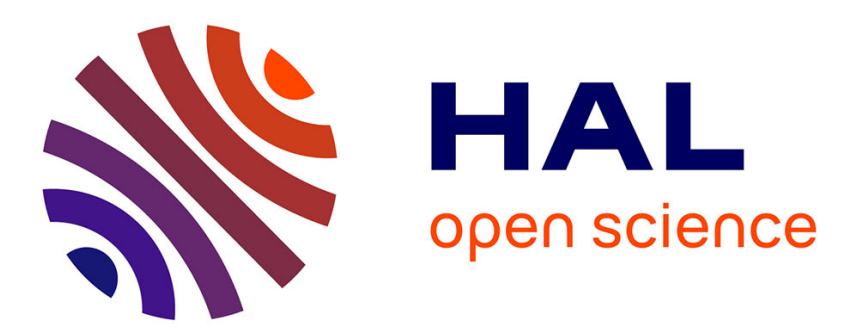

\title{
Numeric and Certified Isolation of the Singularities of the Projection of a Smooth Space Curve
}

\author{
Rémi Imbach, Guillaume Moroz, Marc Pouget
}

\section{To cite this version:}

Rémi Imbach, Guillaume Moroz, Marc Pouget. Numeric and Certified Isolation of the Singularities of the Projection of a Smooth Space Curve. Proceedings of the 6th International Conferences on Mathematical Aspects of Computer and Information Sciences, Oct 2015, Berlin, Germany. hal01239447

\section{HAL Id: hal-01239447 \\ https://inria.hal.science/hal-01239447}

Submitted on 7 Dec 2015

HAL is a multi-disciplinary open access archive for the deposit and dissemination of scientific research documents, whether they are published or not. The documents may come from teaching and research institutions in France or abroad, or from public or private research centers.
L'archive ouverte pluridisciplinaire HAL, est destinée au dépôt et à la diffusion de documents scientifiques de niveau recherche, publiés ou non, émanant des établissements d'enseignement et de recherche français ou étrangers, des laboratoires publics ou privés. 


\title{
Numeric and Certified Isolation of the Singularities of the Projection of a Smooth Space Curve
}

\author{
Rémi Imbach, Guillaume Moroz, and Marc Pouget \\ INRIA Nancy Grand Est, LORIA laboratory, Nancy, France. \\ firstname.name@inria.fr
}

\begin{abstract}
Let $\mathcal{C}_{P \cap Q}$ be a smooth real analytic curve embedded in $\mathbb{R}^{3}$, defined as the solutions of real analytic equations of the form $P(x, y, z)=$ $Q(x, y, z)=0$ or $P(x, y, z)=\frac{\partial P}{\partial z}=0$. Our main objective is to describe its projection $\mathcal{C}$ onto the $(x, y)$-plane. In general, the curve $\mathcal{C}$ is not a regular submanifold of $\mathbb{R}^{2}$ and describing it requires to isolate the points of its singularity locus $\Sigma$. After describing the types of singularities that can arise under some assumptions on $P$ and $Q$, we present a new method to isolate the points of $\Sigma$. We experimented our method on pairs of independent random polynomials $(P, Q)$ and on pairs of random polynomials of the form $\left(P, \frac{\partial P}{\partial z}\right)$ and got promising results.
\end{abstract}

Keywords: Topology of Projected Real Curve, Topology of Apparent Contour, Singularities Isolation, Topology Computation, Numeric Certified Methods

\section{Introduction}

Consider a smooth analytic curve $\mathcal{C}_{P \cap Q} \subset \mathbb{R}^{3}$ defined by $P(x, y, z)=Q(x, y, z)=$ 0 with $P, Q$ analytic functions, and its projection $\mathcal{C} \subset \mathbb{R}^{2}$ on the $(x, y)$-plane. Computing the topology of $\mathcal{C}$, or computing a graph topologically equivalent to $\mathcal{C}$, requires computing the set $\Sigma$ of its singularities (see 1.2 for a rigorous definition). In a second step, the study of the complement of $\Sigma$ allows one to recover the topology of the curve. This fundamental problem arises in fields such as mechanical design, robotics and biology. A specific case of interest is when $Q=P_{z}$ (where $P_{z}$ is the partial derivative $\frac{\partial P}{\partial z}$ ). In this case, the curve $\mathcal{C}$ is the apparent contour of the surface $P(x, y, z)=0$. This case has been intensively studied and extended in the framework of the catastrophe theory (see [10] and references therein). Moreover, determining the topology of a projection of a space curve is an important step to compute its topology $[7,11]$. Similarly determining the topology of the apparent contour of a surface is an important step to compute its topology $[1,5]$.

The goal of this paper is to take advantage of the specific structure of the singularities $\Sigma$ and to propose a characterization allowing to isolate them efficiently. Since we do not restrict our work to the case $P=P_{z}=0$, we also give 
a mathematical description of the types of singularities arising in the projection of curves defined by $P=Q=0$ under some generic assumptions.

Our approach to isolating the singularities $\Sigma$ is to construct a new system so-called ball system, the roots of which are in a one-to-one correspondence with the points of $\Sigma$. As shown with experimental results, this system suits numerical certified solvers such as subdivision methods or homotopy solvers in the polynomial case.

The rest of the paper is organized as follows. Section 2 classifies the singularities of $\mathcal{C}$ and relates them to the points where the projection $\Pi_{x y}$ is not a diffeomorphism. The construction of the ball system and a proof of regularity of its solutions are exhibited in Section 3. Section 4 is dedicated to experiments. The rest of this section presents previous and related works, and gives explicitly the assumptions on $P$ and $Q$ for our method.

\subsection{Previous Works}

State-of-the-art symbolic methods that compute topology of real plane curves defined by polynomials are closely related to bivariate system solving. Many methods use resultant and sub-resultant theory to isolate critical points, see for instance the book chapter [24] and references within. There are some alternatives, using for instance Gröbner bases and rational univariate representations $[28,6]$.

Numerical methods can be used together with interval arithmetic to compute and certify the topology of a non-singular curve when the interest area is a compact subset of the plane $[20,16,27]$. However they fail near any singular point of the curve. Isolating singularities of a plane curve $f(x, y)=0$ with a numerical method is a challenge since it is described by the non-square system $f=f_{x}=$ $f_{y}=0$, and singularities are not necessarily regular solutions of this system. The latter system can be translated into a square system using combinations of its equations with first derivatives [8], and non-regular solutions can be handled through deflation systems (see for instance $[12,26,18,19,13,3]$ ), but the resulting systems are usually still overdetermined or contain spurious solutions.

Such systems are usually solved with symbolic bivariate solvers relying on Gröbner bases or rational univariate representations [28,6]. Well determined systems which solutions are regular can be handled by numerical approaches. Classical homotopy solvers [22] find all complex solutions of latter systems when their equations are polynomials. Subdivision methods [21,25,29] are numeric certified approaches to find all real solutions lying in an initial bounded domain of a system of analytic equations. When the latter are polynomial, these approaches can be extended to unbounded initial domains $[29,25]$.

Starting with the work of Whitney [30], the catastrophe theory was developed to classify the singularities arising while deforming generic mappings (see $[2,10]$ for example). From an algorithmic point of view, the authors of [9] use elements of the catastrophe theory to derive an algorithm isolating the singularities arising in mappings from $\mathbb{R}^{2}$ to $\mathbb{R}^{2}$.

More specifically, the problem of isolating the singularities of the projection of a generic algebraic space curve was investigated in [15]. The authors use 
resultant and sub-resultant theory to represent $\Sigma$ as the solutions of a regular bivariate system suited to a branch and bound solving approach. In Section 4 we compare this approach with the new approach proposed in this article.

\subsection{Notations and Assumptions}

In the following, $\mathcal{C}_{P \cap Q}$ denotes the curve defined as the zero set of the real analytic functions $P(x, y, z)$ and $Q(x, y, z)$ and $B_{0}$ is an open subset of $\mathbb{R}^{2}$. We will denote by $\Pi_{x y}$ the projection from $\mathcal{C}_{P \cap Q}$ to the $(x, y)$-plane, and by $\mathcal{C}$ the projection $\Pi_{x y}\left(\mathcal{C}_{P \cap Q}\right)$.

Regular points and $A_{k}^{ \pm}$singularities. A point $p$ of the curve $\mathcal{C}$ is regular if there is a small neighborhood $U$ of $p$ in $\mathbb{R}^{2}$ such that $\mathcal{C} \cap U$ is a regular submanifold of $\mathbb{R}^{2}$. Otherwise it is singular. A singular point $p$ of a curve $\mathcal{C}$ is of type $A_{k}^{ \pm}$if and only if $\mathcal{C}$ is equal to the solutions of the equation $x^{2} \pm y^{k+1}=0$ on a neighborhood $U$ of $p$, up to a diffeomorphism from $U \subset \mathbb{R}^{2}$ to $V \subset \mathbb{R}^{2}$ ([2, $\S 9.8]$ ). Remark that those are not the only type of singularities that can appear on a plane curve. Notice that the types $A_{2 k}^{+}$and $A_{2 k}^{-}$are equivalent and simply denoted by $A_{2 k}$. We will call node a singularity of type $A_{1}^{-}$or equivalently a transverse intersection of two real curve branches. We also call cusp a singularity of type $A_{2 k}$ and ordinary cusp the singularity $A_{2}$. With this notation, a point $p$ of $\mathcal{C}$ is regular if and only if it is of type $A_{0}$.

In Section 2, we will describe the types of singularities of $\mathcal{C}$ assuming that :

$\left(A_{1}\right)$ The curve $\mathcal{C}_{P \cap Q}$ is smooth above $B_{0}$.

$\left(A_{2}\right)$ For any $(\alpha, \beta)$ in $B_{0}$, the system $P(\alpha, \beta, z)=Q(\alpha, \beta, z)=0$ has at most 2 real roots counted with multiplicities.

$\left(A_{3}\right)$ There is at most a discrete set of points $(\alpha, \beta)$ in $B_{0}$ such that $P(\alpha, \beta, z)=$ $Q(\alpha, \beta, z)=0$ has 2 real roots counted with multiplicities.

$\left(A_{4}\right) \Pi_{x y}$ is a proper map from $\mathcal{C}_{P \cap Q} \cap\left(B_{0} \times \mathbb{R}\right)$ to its image, that is the inverse image of a compact subset is compact.

Then in Section 3, we will introduce the system of analytic equations that we will use to compute the singularities of $\mathcal{C}$. The solutions of this system will be regular under the following additional assumption:

$\left(A_{5}\right)$ The singularities of the curve $\mathcal{C}$ are either nodes or ordinary cusps.

Notice that Thom Transversality Theorem implies that $\left(A_{1}\right),\left(A_{2}\right),\left(A_{3}\right)$ and $\left(A_{5}\right)$ hold for generic analytic maps $P, Q$ defining $\mathcal{C}_{P \cap Q}$ (see [10, Th. 3.9.7 and $\S 4.7])$, and $\left(A_{4}\right)$ holds at least for generic polynomial maps. If we assume only that the curve is smooth (assumption $\left(A_{1}\right)$ ), it would be interesting to prove that all the other assumptions hold after a generic linear change of coordinates.

If $P, Q$ are polynomials, a semi-algorithm checking these conditions is given in [15, Semi-Algo. 1]. Otherwise when $P, Q$ are analytic maps, the latter semialgorithm can be adapted only when $B_{0}$ is bounded. 


\section{Description of the Singularity Locus $\Sigma$}

The different types of singularities of a plane curve have been classified in [2] for example. We describe in this section the types of singularities that can arise on the curve $\mathcal{C}$ under the Assumptions $\left(A_{1}\right)-\left(A_{4}\right)$, and we relate those singularities with the projection mapping $\Pi_{x y}$. More precisely, using Arnold's notation recalled below, we show that under the Assumptions $\left(A_{1}\right)-\left(A_{4}\right)$, the singularities of $\mathcal{C}$ are of type $A_{k}^{ \pm}$(Lemma 2 and Corollary 1$)$. Moreover, we show that a singular point of $\mathcal{C}$ is either a critical value of $\Pi_{x y}$, or the image of two distinct points of $\mathcal{C}_{P \cap Q}$ by $\Pi_{x y}$.

Singularities of $\mathcal{C}$ and critical points of $\Pi_{x y}$. The critical points of $\Pi_{x y}$ are the points of $\mathcal{C}_{P \cap Q}$ where the tangent to the curve is vertical, i.e. aligned with the $z$-axis. Assuming that the conditions $\left(A_{1}\right)-\left(A_{4}\right)$ are satisfied by the curve $\mathcal{C}_{P \cap Q}$, we show that for $p$ a point on the curve $\Pi_{x y}\left(\mathcal{C}_{P \cap Q}\right)$ :

1. if $p$ is a critical point of $\Pi_{x y}$, then it is a cusp point of $\mathcal{C}$ (singularity of type $\left.A_{2(k+1)}\right)$

2. if $p$ is the image of two distinct points of $\mathcal{C}_{P \cap Q}$, then it is a singularity of type $A_{2 k+1}^{-}$;

3. otherwise, it is a regular point.

In particular, this implies that a point $p$ is singular if and only if it is a critical value of $\Pi_{x y}$ or it has two antecedents by $\Pi_{x y}$.

Lemma 1. Let $p$ be a point of $\mathcal{C}$. If $p$ is not a critical value of $\Pi_{x y}$ and $\Pi_{x y}^{-1}(p)$ has only one antecedent, then $p$ is a regular point of $\mathcal{C}$.

Proof. For $U$ an open set of $\mathbb{R}^{2}$, we will denote by $\Pi_{x y}^{U}$ the restriction of $\Pi_{x y}$ to $\mathcal{C}_{P \cap Q} \cap \Pi_{x y}^{-1}(U)$. Since $p$ is not a critical value of $\Pi_{x y}$, there exists a neighborhood $U$ of $p$ such that $U$ does not contain any critical value of $\Pi_{x y}$, such that $\Pi_{x y}^{U}$ is an immersion. Then, since $p$ has a unique antecedent, $\left(A_{3}\right)$ ensures that there is a neighborhood $V$ of $p$ such that $\Pi_{x y}^{V}$ is a homeomorphism. Thus $\Pi_{x y}^{U \cap V}$ is an embedding and $p$ is a regular point.

Lemma 2. Let $p$ be a point of $\mathcal{C}$. If $p$ has two antecedents by $\Pi_{x y}$, then $p$ is a singularity of $\mathcal{C}$ of type $A_{2 k+1}^{-}$with $k \geq 0$.

Proof. If $\Pi_{x y}^{-1}(p)$ contains more than one antecedent of $p$, then $\left(A_{2}\right)$ implies that $p$ has exactly two antecedents $q_{u}$ and $q_{v}$. Since $\Pi_{x y}$ is proper by Assumption $\left(A_{4}\right)$ and $\mathcal{C}_{P \cap Q}$ is smooth by Assumption $\left(A_{1}\right)$, for a small enough neighborhood $U$ of $p, \Pi_{x y}^{-1}(U)$ is bounded and is the union of two smooth connected branches of $\mathcal{C}_{P \cap Q}$. And $\left(A_{3}\right)$ implies that in a small enough neighborhood of $p, p$ is the only point with two antecedents. Let $u=\left(u_{x}, u_{y}, u_{z}\right)$ and $v=\left(v_{x}, v_{y}, v_{z}\right)$ be the two vectors tangent to $\mathcal{C}_{P \cap Q}$ at the antecedents $q_{u}$ and $q_{v}$ of $p$. Assumption $\left(A_{2}\right)$ implies that neither $u$ nor $v$ are vertical, hence $\tilde{u}=\left(u_{x}, u_{y}\right)$ and $\tilde{v}=\left(v_{x}, v_{y}\right)$ are non-zero vectors of $\mathbb{R}^{2}$. We now distinguish two cases. 
First, $\tilde{u}$ and $\tilde{v}$ are independent vectors. In this case, the mapping $\left(\begin{array}{l}X \\ Y\end{array}\right)=$ $\left(\begin{array}{ll}u_{x} & u_{y} \\ v_{x} & v_{y}\end{array}\right)^{-1} \cdot\left(\begin{array}{l}x \\ y\end{array}\right)$ is a diffeomorphic change of coordinates. Moreover $\left(\begin{array}{c}P_{X}\left(q_{u}\right) \\ Q_{X}\left(q_{u}\right)\end{array}\right)=$ $\left(\begin{array}{l}0 \\ 0\end{array}\right)$ and $\left(\begin{array}{c}P_{Y}\left(q_{u}\right) \\ Q_{Y}\left(q_{u}\right)\end{array}\right) \neq\left(\begin{array}{l}0 \\ 0\end{array}\right)$. Thus by the analytic implicit function theorem, there exists an analytic function $f: \mathbb{R} \mapsto \mathbb{R}$ such that $Y=f(X)$ and $f(0)=f^{\prime}(0)=0$ such that the projection of the branch at $q_{u}$ has an equation of the form $Y=$ $X^{2} \tilde{f}(X)$. Symmetrically, the projection of the branch at $q_{v}$ has an equation of the form $X=Y^{2} \tilde{g}(Y)$. Thus, up to a diffeomorphism of $\mathbb{R}^{2}$, the curve $\mathcal{C}$ around $p$ has an equation of the form $\left(Y-X^{2} \tilde{f}(X)\right)\left(X-Y^{2} \tilde{g}(Y)\right)=0$, or equivalently $\left(X+Y-X^{2} \tilde{f}(X)-Y^{2} \tilde{g}(Y)\right)^{2}-\left(X-Y-X^{2} \tilde{f}(X)+Y^{2} \tilde{G}(Y)\right)^{2}=0$. That is, $p$ is a singularity of type $A_{1}^{-}$, also called a node.

In the case where $\tilde{u}$ and $\tilde{v}$ are co-linear, we follow the same approach, using this time the diffeomorphic change of coordinate $\left(\begin{array}{c}X \\ Y\end{array}\right)=\left(\begin{array}{cc}u_{x} & u_{y} \\ -u_{y} & u_{x}\end{array}\right)^{-1} \cdot\left(\begin{array}{l}x \\ y\end{array}\right)$. Moreover $\left(\begin{array}{c}P_{X}\left(q_{u}\right) \\ Q_{X}\left(q_{u}\right)\end{array}\right)=\left(\begin{array}{l}0 \\ 0\end{array}\right)$. As in the previous case, we use the analytic implicit function theorem at $q_{u}$ and $q_{v}$, and we conclude that there exist two analytic functions $f$ and $g$ such that on a neighborhood of $p$, the curve $\mathcal{C}$ is given by the equation $\left(Y-X^{2} f(X)\left(Y-X^{2} g(X)\right)=0\right.$. That can be rewritten as $\left(2 Y-X^{2}(f(X)+g(X))\right)^{2}-X^{4}(g(X)-f(X))^{2}=0$. Assumption $\left(A_{3}\right)$ ensures that the projections of the 2 branches have only one common point, such that $g(X)-f(X)$ does not vanish identically. Then, denoting by $k$ the valuation of $f(X)-g(X), p$ is a singularity of type $A_{2 k+3}^{-}$.

Finally, if $p$ is a critical value of $\Pi_{x y}$ we use Arnold's classification of singularities and prove that $p$ is a singular point of type $A_{2(k+1)}$ with $k \geq 0$.

Lemma 3. Assume that the curve $\mathcal{C}_{P \cap Q}$ satisfies $\left(A_{1}\right)-\left(A_{3}\right)$. Let $q$ be a critical point of $\Pi_{x y}$. Then, there exists a neighborhood $U$ of $q$ and an invertible $2 \times 2$ matrix $M$ of real analytic functions such that:

$$
\left(\begin{array}{l}
P \\
Q
\end{array}\right)=M \cdot\left(\begin{array}{c}
X-Z^{3+2 k} \\
Y-Z^{2}
\end{array}\right) \circ \Phi(x, y, z)
$$

where $\Phi:(x, y, z) \mapsto(\phi(x, y), \psi(z))$ is a diffeomorphism and $k$ is a natural integer.

Corollary 1. Let $p$ be a point of $\mathcal{C}$. If $p$ is a critical value of $\Pi_{x y}$, then $p$ is a cusp of $\mathcal{C}$ of type $A_{2(k+1)}$ with $k \geq 0$.

Proof (of the corollary). Let $q$ be the critical point associated to $p$ and denote $\pi_{x y}$ the projection from $\mathbb{R}^{3}$ to $\mathbb{R}^{2}$. First we show that it is sufficient to study the behavior of $\mathcal{C}_{P \cap Q}$ in a neighborhood of $q$ to describe the curve $\mathcal{C}$ in a neighborhood of $p$. Indeed, Assumptions $\left(A_{2}\right)$ and $\left(A_{4}\right)$ imply that above a small enough neighborhood of $p$, the curve $\mathcal{C}_{P \cap Q}$ has a unique connected branch. In particular for any neighborhood $U$ of the critical point $q$ there exists a neighborhood $V \subset U$ such that $\pi_{x y}(V) \cap \mathcal{C} \subset \Pi_{x y}\left(U \cap \mathcal{C}_{P \cap Q}\right)$.

Then, Lemma 3 shows that there exists a neighborhood $U$ of $q$ and a diffeomorphism $\phi$ from $\pi_{x y}(U) \subset \mathbb{R}^{2}$ to $V \subset \mathbb{R}^{2}$ a neighborhood of $(0,0)$ such that 
$\phi\left(\Pi_{x y}\left(\mathcal{C}_{P \cap Q} \cap U\right)\right)=\left\{(X, Y) \in V \mid X^{2}-Y^{3+2 k}\right\}$. In particular, $p$ is a singularity of type $A_{2(k+1)}$ with $k \geq 0$, that is a cusp.

Proof (of Lemma 3). This lemma is essentially a consequence of the analytic implicit function theorem, combined with our assumptions. First, $q$ is a critical point thus $\mathcal{C}_{P \cap Q}$ has a vertical tangent at $q$, up to a translation, we assume $q=(0,0,0)$. Since $\mathcal{C}_{P \cap Q}$ is non-singular (Assumption $\left(A_{1}\right)$ ), the matrix $\left(\begin{array}{cc}P_{x}(q) & P_{y}(q) \\ Q_{x}(q) & Q_{y}(q)\end{array}\right)$ is invertible. Using the analytic implicit function theorem ([17] or [10, Corollary 2.7.3]), there exist two real analytic functions $f, g$ from $\mathbb{R}$ to $\mathbb{R}$ such that $P(f(z), g(z), z)=Q(f(z), g(z), z)=0$ on a small enough neighborhood of 0 . In particular, letting $X:=x-f(z)$ and $Y=y-g(z)$ we have $P=P(X+f(z), Y+g(z), z)$ and $Q=Q(X+f(z), Y+g(z), z)$. Using Hadamard's lemma ([10, Proposition 4.2.3]), there exist real analytic functions $a, b, c, d$ such that $P=a \cdot X+b \cdot Y$ and $Q=c \cdot X+d \cdot Y$. Moreover, since $\left(\begin{array}{cc}P_{x}(q) & P_{y}(q) \\ Q_{x}(q) & Q_{y}(q)\end{array}\right)$ is invertible, the matrix $\left(\begin{array}{ll}a(q) & b(q) \\ c(q) & d(q)\end{array}\right)$ is also invertible. Let $M_{1}$ be the inverse of $\left(\begin{array}{ll}a & b \\ c & d\end{array}\right)$ on a small enough neighborhood of $q$. Then we have:

$$
\left(\begin{array}{c}
\tilde{P} \\
\tilde{Q}
\end{array}\right):=\left(\begin{array}{c}
x-f(z) \\
y-g(z)
\end{array}\right)=M_{1} \cdot\left(\begin{array}{l}
P \\
Q
\end{array}\right)
$$

Moreover, since the curve has a vertical tangent at $q$, we have $f_{z}(0)=g_{z}(0)=0$. And according to Assumption $\left(A_{2}\right)$, either $f_{z z}(0)$ or $g_{z z}(0)$ is not zero. Without restriction of generality, assume $\mu:=g_{z z}(0) \neq 0$. Up to a scale of the variable $z$, we can assume that $\mu=2$. Thus, there exist analytic functions $u, v$ such that $f$ and $g$ are of the form:

$$
\begin{aligned}
& f(z)=z^{2} u(z) \\
& g(z)=z^{2}(1+z v(z)) .
\end{aligned}
$$

Letting $\psi: z \mapsto Z:=z \sqrt{1+z v(z)}$, we have $\tilde{Q}\left(x, y, \psi^{-1}(Z)\right)=y-Z^{2}=0$. In particular, the function $\tilde{P}=x-z^{2} u(z)$ can be rewritten as $\tilde{P}\left(x, y, \psi^{-1}(Z)\right)=x-$ $Z^{2}\left(s\left(Z^{2}\right)+Z t\left(Z^{2}\right)\right)$ with $s$ and $t$ two real analytic functions. Note that $t$ cannot have all its derivatives vanishing at 0 since otherwise there would be a strictly positive dimensional set of points with two or more antecedents, contradicting Assumption $\left(A_{3}\right)$. Let $k \in \mathbb{N}$ be the valuation of $t$, i.e. its first non vanishing derivative at 0 . Then, there exists $t^{\prime}$ an analytic function such that $t\left(Z^{2}\right)$ is of the form $Z^{2 k}\left(\eta+Z^{2} t^{\prime}\left(Z^{2}\right)\right)$. The function $\tilde{P}\left(x, y, \psi^{-1}(Z)\right)$ is of the form $x-Z^{2}\left(s\left(Z^{2}\right)+Z^{1+2 k}\left(\eta+Z^{2} t^{\prime}\left(Z^{2}\right)\right)\right)$. Using $\tilde{Q}$ to substitute $\psi(z)^{2}$ by $y$ in $\tilde{P}$, there exists a matrix $M_{2}:=\left(\begin{array}{cc}1 & e \\ 0 & 1\end{array}\right)$ where $e$ is an analytic function, such that:

$$
\left(\begin{array}{c}
x-\frac{s(y)}{y}-\psi(z)^{3+2 k}(\eta+y t(y)) \\
y-\psi(z)^{2}
\end{array}\right)=M_{2} \cdot M_{1} \cdot\left(\begin{array}{l}
P \\
Q
\end{array}\right) .
$$


Finally, with:

$$
\begin{aligned}
\phi(x, y) & =\left(\frac{x-\frac{s(y)}{y}}{\eta+y t(y)}, y\right) \\
\psi(z) & =z \sqrt{1+z v(z)} \\
M & =M_{1}^{-1} \cdot M_{2}^{-1} \cdot\left(\begin{array}{rr}
\frac{1}{\eta+y t(y)} & 0 \\
0 & 1
\end{array}\right)
\end{aligned}
$$

we recover (1).

\section{Modeling System}

Following the result of Section 2, a naive approach to represent the singularities $\Sigma$ of $\mathcal{C}$ is to use the two following systems.

1. For $\left(x, y, z_{1}, z_{2}\right) \in B_{0} \times \mathbb{R}^{2}$ :

$$
P\left(x, y, z_{1}\right)=P\left(x, y, z_{2}\right)=Q\left(x, y, z_{1}\right)=Q\left(x, y, z_{2}\right)=0 \text { and } z_{1} \neq z_{2} .
$$

2. For $(x, y, z) \in B_{0} \times \mathbb{R}$ :

$$
P(x, y, z)=Q(x, y, z)=P_{z}(x, y, z)=Q_{z}(x, y, z)=0 .
$$

However, the first system is numerically unstable near the set $z_{1}=z_{2}$ and the second one is over-determined. Instead, we will introduce an unified system. First we define the operators that will be used to construct our system.

\subsection{Ball System}

Definition 1. Let $A(x, y, z)$ be a real analytic function. We denote by $S . A$ and D.A the functions:

$$
\begin{aligned}
& \text { S.A(x,y,c, } \left.r_{2}\right)= \begin{cases}\frac{1}{2}\left(A\left(x, y, c+\sqrt{r_{2}}\right)+A\left(x, y, c-\sqrt{r_{2}}\right)\right) & \text { if } r_{2}>0 \\
A(x, y, c) & \text { if } r_{2}=0 \\
\frac{1}{2}\left(A\left(x, y, c+i \sqrt{-r_{2}}\right)+A\left(x, y, c-i \sqrt{-r_{2}}\right)\right) & \text { if } r_{2}<0\end{cases} \\
& D . A\left(x, y, c, r_{2}\right)=\left\{\begin{array}{lr}
\frac{1}{2 \sqrt{r_{2}}}\left(A\left(x, y, c+\sqrt{r_{2}}\right)-A\left(x, y, c-\sqrt{r_{2}}\right)\right) & \text { if } r_{2}>0 \\
A_{z}(x, y, c) & \text { if } r_{2}=0 \\
\frac{1}{2 \sqrt{-r_{2}}}\left(A\left(x, y, c+i \sqrt{-r_{2}}\right)-A\left(x, y, c-i \sqrt{-r_{2}}\right)\right) & \text { if } r_{2}<0
\end{array} .\right.
\end{aligned}
$$

By abuse of notation, if $M$ is a matrix of real analytic functions, S.M and D.M denote the matrices with the operator applied on each entry. 
If $A$ is a real analytic function, then $S . A$ and $D . A$ are also real analytic functions (see Lemma 6). This allows us to introduce the so-called ball system that we will use to compute $\Sigma$. In this system we map two solutions $\left(x, y, z_{1}\right)$ and $\left(x, y, z_{2}\right)$ of $P=Q=0$ (or $P=P_{z}=0$ ) to their center $(x, y, c)$ and the square of their radius $r_{2}=r^{2}$, with $r=\left|z_{1}-c\right|=\left|z_{2}-c\right|$. Figure 1 illustrates this mapping for singularities of the apparent contour of a torus. Its left part shows the surface $P=0$, its set of $z$-critical points $\mathcal{C}_{P \cap P_{z}}$ and the apparent contour $\mathcal{C}=\Pi_{x y}\left(\mathcal{C}_{P \cap P_{z}}\right)$. Its right part shows, for nodes and ordinary cusp singularities, their respective antecedents by $\Pi_{x y}$, centers $c$ and radii $r$.
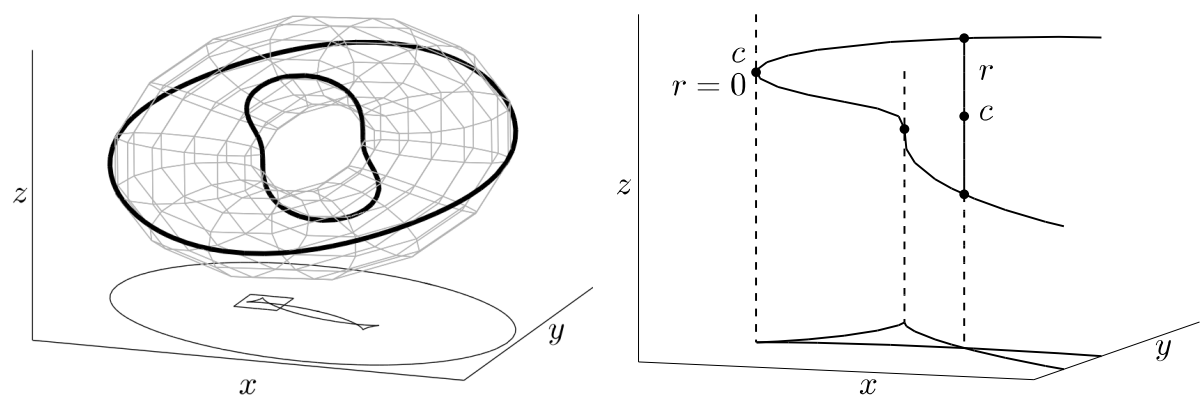

Fig. 1. Left: a torus, in bold line its set of $z$-critical points, its apparent contour, and the zoom zone corresponding to the right figure. Right: a detail, with antecedents, centers and radius corresponding to singularities.

Lemma 4. Let $\mathcal{S}$ be the set of solutions of the so-called ball system:

$$
\left\{\begin{array}{l}
S . P\left(x, y, c, r_{2}\right)=0 \\
S . Q\left(x, y, c, r_{2}\right)=0 \\
D . P\left(x, y, c, r_{2}\right)=0 \\
D . Q\left(x, y, c, r_{2}\right)=0
\end{array}\right.
$$

in $B_{0} \times \mathbb{R} \times \mathbb{R}^{+}$. Then $\Pi_{x y}^{\prime}(\mathcal{S})=\Sigma$, where $\Pi_{x y}^{\prime}$ is the projection from $\mathbb{R}^{4}$ to the $(x, y)$-plane.

Proof. According to Section 2, the singularity locus of $\mathcal{C}$ is exactly the union of the critical values of $\Pi_{x y}$ and of the points that have several antecedents. They correspond respectively to the solutions of $\mathcal{S}$ such that $r=0$ and such that $r>0$.

One of the main advantage of this system is that its solutions are regular when the condition $\left(A_{5}\right)$ is satisfied, and thus can be solved using certified numerical algorithms such as homotopy or subdivision methods (see Section 4). 
Lemma 5. Under the Assumptions $\left(A_{1}\right)-\left(A_{4}\right)$, all the solutions of the system $S . P=S . Q=D . P=D . Q=0$ in $B_{0} \times \mathbb{R} \times \mathbb{R}^{+}$are regular if and only if $\left(A_{5}\right)$ is satisfied.

The next subsection is dedicated to the proof of this lemma.

\subsection{Regularity Condition}

Lemma 6. If $A$ is a real analytic function, then S.A and D.A are real analytic functions. Moreover, the derivatives of $S . A$ with respect to $x, y, c, r_{2}$ are respectively $S . A_{x}, S . A_{y}, S . A_{z}, \frac{1}{2} D . A_{z}$. The derivative of D.A with respect to $x, y, c, r_{2}$ are respectively D. $A_{x}, D . A_{y}, D . A_{z}$ and $\frac{S . A_{z}-D . A}{2 r_{2}}$ if $r_{2}>0$ and $\frac{1}{6} A_{z z z}$ if $r_{2}=0$.

Proof. First, on a neighborhood of $r_{2}>0, S . A$ and D.A are compositions of analytic functions, and thus are analytic. Likewise, for $r_{2}<0, S . A$ and D.A are analytic functions, and all the coefficients of their series expansions are real, thus they are real valued analytic functions. Finally, on a neighborhood of $(x, y, c, 0)$, if $A(x, y, c+r)=\sum_{n=0}^{\infty} a_{n}(x, y, c) r^{n}$, the series expansions of $S . A$ and D.A for $r_{2}<0, r_{2}=0$ and $r_{2}>0$ coincide as:

$$
\begin{aligned}
& S . A\left(x, y, c, r_{2}\right)=\sum_{n=0}^{\infty} a_{2 n}(x, y, c) r_{2}^{n} \\
& D . A\left(x, y, c, r_{2}\right)=\sum_{n=0}^{\infty} a_{2 n+1}(x, y, c) r_{2}^{n} .
\end{aligned}
$$

Thus S.A and D.A are analytic functions. The expressions of their derivatives follow from the formulas.

Lemma 7. If $\psi: U \subset \mathbb{R}^{3} \mapsto V \subset \mathbb{R}^{3}$ is an analytic diffeomorphism of the form $\psi(x, y, z)=\left(\psi_{1}(x, y), \psi_{2}(x, y), \psi_{3}(x, y, z)\right)$, so-called triangular, then the mapping:

$$
S D . \psi:\left(x, y, c, r_{2}\right) \mapsto\left(\psi_{1}(x, y), \psi_{2}(x, y), S . \psi_{3}\left(x, y, c, r_{2}\right), r_{2}\left(D . \psi_{3}\left(x, y, c, r_{2}\right)\right)^{2}\right)
$$

is a real analytic diffeomorphism from $\left\{\left(x, y, c, r_{2}\right) \in \mathbb{R}^{3} \times \mathbb{R}^{+} \mid\left(x, y, c+\sqrt{r_{2}}\right) \in\right.$ $U\}$ to $\left\{\left(X, Y, C, R_{2}\right) \in \mathbb{R}^{3} \times \mathbb{R}^{+} \mid\left(X, Y, C+\sqrt{R_{2}}\right) \in V\right\}$.

Moreover, if $A: \mathbb{R}^{3} \rightarrow \mathbb{R}$ is an analytic map, we have:

$$
\begin{aligned}
S .(A \circ \psi) & =(S . A) \circ(S D . \psi) \\
D .(A \circ \psi) & =(D . A) \circ(S D . \psi) \times D . \psi_{3}
\end{aligned}
$$

Proof. According to the previous lemma, $S D . \psi$ is analytic. Moreover, since $\psi^{-1}$ is analytic, $S D .\left(\psi^{-1}\right)$ is also analytic. Assuming that the inequalities at the end of the lemma are correct, we can use them to check that $S D .\left(\psi^{-1}\right) \circ S D . \psi$ is the identity by developing the formula. Such that $S D . \psi$ is a diffeomorphism.

To prove the final identities of the lemma, let $\left(X, Y, C, R_{2}\right)=S D \cdot \psi\left(x, y, c, r_{2}\right)$. We can observe that $\psi_{3}\left(x, y, c+\sqrt{r_{2}}\right)=C+\sqrt{R_{2}}$ and $\psi_{3}\left(x, y, c-\sqrt{r_{2}}\right)=C-\sqrt{R_{2}}$ 
by expanding $S . \psi_{3}+\sqrt{r_{2}\left(D . \psi_{3}\right)^{2}}$ and $S . \psi_{3}-\sqrt{r_{2}\left(D . \psi_{3}\right)^{2}}$. Using these formula, we can deduce the identities by expanding the right and left hand side of the equalities.

Lemma 8. Let $P, Q$ be two analytic functions from $U \subset \mathbb{R}^{3}$ to $\mathbb{R}$ and assume that there exist two analytic functions $\tilde{P}, \tilde{Q}$, a $2 \times 2$ invertible matrix of analytic functions and a triangular diffeomorphism $\phi: U \rightarrow V \subset \mathbb{R}^{3}$ such that $\left(\begin{array}{c}P \\ Q\end{array}\right)=$ $M \cdot\left(\begin{array}{c}\tilde{P} \\ \tilde{Q}\end{array}\right) \circ \phi$. Then we have:

$$
\left(\begin{array}{c}
S . P \\
S . Q \\
D . P \\
D . Q
\end{array}\right)=\underbrace{\left(\begin{array}{cc}
S . M & r_{2} D \cdot \phi_{3} D \cdot M \\
D . M & D \cdot \phi_{3} S \cdot M
\end{array}\right)}_{T}\left(\begin{array}{c}
S . \tilde{P} \\
S . \tilde{Q} \\
D . \tilde{P} \\
D \cdot \tilde{Q}
\end{array}\right) \circ S D \cdot \phi
$$

where the matrix $T$ is invertible, of inverse $\tilde{T}:=\left(\begin{array}{cc}S \cdot M^{-1} & r_{2} D \cdot M^{-1} \\ D \cdot M^{-1} / D \cdot \phi_{3} & S \cdot M^{-1} / D \cdot \phi_{3}\end{array}\right)$.

Proof. First, using the identity $a b+c d=\frac{1}{2}(a+c)(b+d)+\frac{1}{2}(a-c)(b-d)$, we can deduce :

$$
\left(\begin{array}{c}
S . P \\
S . Q \\
D . P \\
D . Q
\end{array}\right)=\left(\begin{array}{cc}
S . M & r_{2} D \cdot M \\
D . M & S . M
\end{array}\right)\left(\begin{array}{c}
S \cdot(\tilde{P} \circ \phi) \\
S \cdot(\tilde{Q} \circ \phi) \\
D \cdot(\tilde{P} \circ \phi) \\
D \cdot(\tilde{Q} \circ \phi)
\end{array}\right)
$$

Finally, expanding the operators in the righthand side vector using the formula in Lemma 7, we prove the desired identity. Finally, since $\phi$ is a triangular diffeomorphism, we can use the formula of Lemma 7 with $A=\phi_{3}^{-1}$ to get $1=D\left(\left(\phi^{-1}\right)_{3} \circ \phi\right)=D \cdot\left(\phi^{-1}\right)_{3} \circ S D \cdot \phi \times D \cdot \phi_{3}$. In particular, D. $\phi_{3}$ is never 0 and $\tilde{T}$ is well defined. Expanding $\tilde{T} \cdot T$, we get the identity, such that $\tilde{T}$ is the inverse of $T$.

Corollary 2. A point $p$ solution of the system S.P $=S \cdot Q=D \cdot P=D \cdot Q=0$ is regular if and only if the point $S D . \phi(p)$ is regular in the system $S \cdot \tilde{P}=S \cdot \tilde{Q}=$ $D . \tilde{P}=D . \tilde{Q}=0$.

Proof. The claim of the lemma can be verified by developing the product vector. For the corollary, it is sufficient to observe that on a point $p$ solution of the system, the Jacobian matrices satisfy the relation:

$$
\operatorname{Jac}_{p}\left(\begin{array}{c}
S . P \\
S . Q \\
D . P \\
D . Q
\end{array}\right)(p)=T \cdot \operatorname{Jac}_{S D . \phi(p)}\left(\begin{array}{c}
S . \tilde{P} \\
S . \tilde{Q} \\
D . \tilde{P} \\
D . \tilde{Q}
\end{array}\right) \cdot \operatorname{Jac}_{p}(S D . \phi)
$$

We have now all the tools necessary to prove Lemma 5. 
Proof (of Lemma 5). First, let $q$ be a solution of our system with $r_{2}=0$. Then, according to Lemma 3, there exists an invertible matrix $M$ and a triangular diffeomorphism $\phi$ such that on a neighborhood of $q$ we have:

$$
\left(\begin{array}{l}
P \\
Q
\end{array}\right)=M \cdot\left(\begin{array}{c}
X-Z^{3+2 k} \\
Y-Z^{2}
\end{array}\right) \circ \Phi(x, y, z)
$$

Thus, the point $q$ is regular in the ball system if and only if $(0,0,0)$ is regular in the ball system generated by $X-Z^{3+2 k}$ and $Y-Z^{2}$ (Corollary 2). Computing the associated Jacobian matrix, we can check that $q$ is regular if and only if $k=0$, that is, if and only if its projection $p$ is an ordinary cusp.

Now, let $q=\left(x, y, c, r_{2}\right)$ be a solution of the ball system with $r_{2}>0$. In this case $q$ represents two points $q_{1}=\left(x, y, c+\sqrt{r_{2}}\right)$ and $q_{2}=(x, y, c-$ $\sqrt{r_{2}}$ ) of $\mathcal{C}_{P \cap Q}$ with the same projection. According to Lemma 6 the equation $\operatorname{det} J a c_{\left(x, y, c, r_{2}\right)}(S . P, S . Q, D . P, D . Q)=0$ can be written

$$
\left|\begin{array}{lllc}
S . P_{x} & S . P_{y} & S . P_{z} & \frac{D . P_{z}}{2} \\
S . Q_{x} & S . Q_{y} & S . Q_{z} & \frac{D . Q_{z}}{2} \\
D . P_{x} & D . P_{y} & D . P_{z} & \frac{S . P_{z}-D . P}{2 r_{2}} \\
D . Q_{x} & D . Q_{y} & D . Q_{z} & \frac{S \cdot Q_{z}-D . Q}{2 r_{2}}
\end{array}\right|=0
$$

This determinant simplifies using the facts that a) $D \cdot P=D \cdot Q=0$ at the solutions, b) one can multiply lines 3 and 4 by $\sqrt{r_{2}}$ and column 4 by $2 \sqrt{r_{2}}, \mathrm{c}$ ) one can replace lines $\ell_{1}, \ell_{3}$ by $\ell_{1}+\ell_{3}, \ell_{1}-\ell_{3}$ and $\ell_{2}, \ell_{4}$ by $\ell_{2}+\ell_{4}, \ell_{2}-\ell_{4}$. The equation is then equivalent to

$$
\left|\begin{array}{llll}
P_{x}\left(q_{1}\right) & P_{y}\left(q_{1}\right) & P_{z}\left(q_{1}\right) & P_{z}\left(q_{1}\right) \\
Q_{x}\left(q_{1}\right) & Q_{y}\left(q_{1}\right) & Q_{z}\left(q_{1}\right) & Q_{z}\left(q_{1}\right) \\
P_{x}\left(q_{2}\right) & P_{y}\left(q_{2}\right) & P_{z}\left(q_{2}\right) & -P_{z}\left(q_{2}\right) \\
Q_{x}\left(q_{2}\right) & Q_{y}\left(q_{2}\right) & Q_{z}\left(q_{2}\right) & -Q_{z}\left(q_{2}\right)
\end{array}\right|=0
$$

Expending this expression, one can check that it is equivalent to

$$
\left|\begin{array}{l}
P_{y}\left(q_{1}\right) Q_{z}\left(q_{1}\right)-P_{z}\left(q_{1}\right) Q_{y}\left(q_{1}\right) P_{y}\left(q_{2}\right) Q_{z}\left(q_{2}\right)-P_{z}\left(q_{2}\right) Q_{y}\left(q_{2}\right) \\
P_{z}\left(q_{1}\right) Q_{x}\left(q_{1}\right)-P_{x}\left(q_{1}\right) Q_{z}\left(q_{1}\right) P_{z}\left(q_{2}\right) Q_{x}\left(q_{2}\right)-P_{x}\left(q_{2}\right) Q_{z}\left(q_{2}\right)
\end{array}\right|=0
$$

The later expression is equivalent to the condition that projection on the $(x, y)$ plane of the tangent vectors of the $3 \mathrm{D}$ curve $\mathcal{C}_{P \cap Q}$ at the points $q_{1}$ and $q_{2}$ are collinear. Thus in the case where $r_{2}>0$, a solution of the ball system is regular iff it projects to a node.

\section{Experiments}

We propose some quantitative results on the isolation of the singularities of the projection $\mathcal{C}$ of a space real curve $\mathcal{C}_{P \cap Q}$ (or $\mathcal{C}_{P \cap P_{z}}$ in the case of an apparent contour) by solving the ball system proposed in this paper. We consider here that $P$ and $Q$ are polynomials, hence the equations of the ball system are polynomials 
and $\mathcal{C}$ admits at most finitely many singularities in $\mathbb{R}^{2}$. Under our assumptions, the curve $\mathcal{C}^{\prime}$ defined as the resultant of $P$ and $Q$ with respect to $z\left(Q=P_{z}\right.$ in the case of an apparent contour) is the union of $\mathcal{C}$ and a finite set of isolated points. Its singularities can be characterized as real solutions of a bivariate system based on the sub-resultant chain of $P$ and $Q$ (or $P_{z}$ ) (see [15]). We compare the resolution with three state-of-the-art methods of the sub-resultant system, denoted by $\mathcal{S}_{2}$ in what follows, and the ball system $S \cdot P=S \cdot Q=D \cdot P=D \cdot Q=0$ defined in Subsection 3.1, denoted by $\mathcal{S}_{4}$.

Experimental Data are random dense polynomials $P, Q$ generated with degree $d$ and integer coefficients chosen uniformly in $\llbracket-2^{8}, 2^{8} \rrbracket$. Unless explicitly stated, the given running times are averages over five instances for a given degree $d$.

Testing Environment is a $\operatorname{Intel}(\mathrm{R}) \mathrm{Xeon}(\mathrm{R}) \mathrm{CPU} \mathrm{L} 5640 @ 2.27 \mathrm{GHz}$ machine with Linux.

\subsection{Resolution Methods}

Gröbner Basis and Rational Univariate Representations allow one to find all real roots of a system of polynomials. The routine Isolate of the mathematical software Maple implements this approach.

Homotopy Continuation provides all the complex solutions of a system of polynomials and relies on a numerical path-tracking step. Among available opensource software implementing homotopy, we chose Bertini ${ }^{1}$ notably because it handles both double precision (DP) and an Adaptive Multi-Precision (AMP) arithmetics [4]. This is necessary to prevent the loss of solutions in system $\mathcal{S}_{2}$ which coefficients are quotients of big integers (see Table 2).

Subdivision uses interval arithmetic (see $[21,25,29]$ for an introduction) to compute for a given system all its regular solutions lying in an initial open box $B_{0} \subset \mathbb{R}^{n}$. Here $n=2$ for system $\mathcal{S}_{2}$ and $n=4$ for system $\mathcal{S}_{4}$. When $P, Q$ are polynomials, the initial box can be $\mathbb{R}^{n}$ (see [25, p.210] or [29, p.233]). Otherwise, $B_{0}$ is bounded, and the number of singularities is finite. Since we focus on singularities induced by projection of real parts of the curve $\mathcal{C}_{P \cap Q}$ or $\mathcal{C}_{P \cap P_{z}}$, we did only research solutions of the ball system having $r_{2} \geq 0$. We implemented a subdivision solver in $\mathrm{c}^{++}$, using the boost or mpfi interval arithmetic library. The implementation is described with more details in [15].

\subsection{Singularities Isolation: Comments on Tables 1, 2 and 3}

Tables 1, 2 and 3 report the sequential running times (columns t) in seconds to compute the singularities of projection and apparent contour curves, using system $\mathcal{S}_{2}$ or system $\mathcal{S}_{4}$ to represent their singularities.

\footnotetext{
1 https://bertini.nd.edu/
} 
Table 1 shows that for Isolate running times are better when solving system $\mathcal{S}_{2}$, due to its lower number of variables.

Table 2 refers to resolution with Bertini, using DP and AMP arithmetics. In addition to running times, it reports the number of missed solutions (columns Mis. Sols.) when using DP arithmetic. The resolution by homotopy in DP of system $\mathcal{S}_{2}$ is not satisfactory due to the high number of missed solutions. The use of AMP arithmetic resolves this problem: for all systems we tested, all solutions were found. But it induces an important additional cost. System $\mathcal{S}_{4}$ seems better suited to homotopy resolution. In DP arithmetic, fewer solutions are missed and the cost of AMP arithmetic is more acceptable. Notice however that for three examples, a solution was missed both with DP and AMP arithmetic due to the truncation of a path considered as converging to a solution at infinity.

Table 3 reports results obtained with our implementation of subdivision. For a given degree, resolution times are subject to an important variance. For low degrees it is more efficient to solve system $\mathcal{S}_{2}$ than system $\mathcal{S}_{4}$ due to the higher dimension (i.e. 4 instead of 2) of the research space in the latter case. The difference of running times decreases when $d$ increases, due to the size (in terms of degree, number of monomials and bit-size of coefficients) of the resultant and sub-resultant polynomials that have to be evaluated to solve system $\mathcal{S}_{2}$.

Table 1. Isolating singularities of projection and apparent contour curves with the routine Isolate of Maple. Input polynomials have degree $d$. The running times are in seconds.

\begin{tabular}{c|c|c||c|c|} 
& \multicolumn{2}{|c||}{ Projection } & \multicolumn{2}{c|}{ Apparent contour } \\
system $\mathcal{S}_{2}$ & system $\mathcal{S}_{4}$ & system $\mathcal{S}_{2}$ & system $\mathcal{S}_{4}$ \\
$d$ & $\mathrm{t}$ & $\mathrm{t}$ & $\mathrm{t}$ & $\mathrm{t}$ \\
\hline 4 & 1.321 & 4.293 & 0.206 & 0.1874 \\
5 & 26.92 & 100.4 & 5.439 & 6.501 \\
6 & $(\mathrm{a})$ & (a) & 98.59 & 155.8 \\
7 & (a) & (a) & (a) & (a)
\end{tabular}

(a) Fails with error

\section{Conclusion}

Given an analytic curve $\mathcal{C}_{P \cap Q}$ satisfying some specific generic assumptions, we have described the different possible types of singularities $\Sigma$ of its projection $\mathcal{C}=$ $\Pi_{x y}\left(\mathcal{C}_{P \cap Q}\right)$. Moreover we have shown that these singularities can be computed as the regular solutions of a new so-called ball system.

Even if our characterization increases the number of variables of the system to solve in order to compute $\Sigma$, we have shown with experimental results that 
Table 2. Isolating singularities of projection and apparent contour curves with Bertini using DP and AMP arithmetic. Input polynomials have degree $d$. The running times are in seconds.

\begin{tabular}{|c|c|c|c|c|c|c|c|c|c|c|c|}
\hline \multirow[b]{3}{*}{$d$} & \multicolumn{5}{|c|}{ Projection } & \multicolumn{6}{|c|}{ Apparent contour } \\
\hline & & stem $\mathcal{S}_{2}$ & & & ystem $\mathcal{S}_{4}$ & & stem $\mathcal{S}_{2}$ & & & ystem $\mathcal{S}_{4}$ & \\
\hline & $\mathrm{t}$ & Mis. Sols. & & $\mathrm{t}$ & Mis. Sols. & $\mathrm{t}$ & Mis. Sols. & & $\mathrm{t}$ & Mis. Sols. & \\
\hline 4 & 0.864 & 0 & & 1.376 & $\begin{array}{ll}1 & (\mathrm{c})\end{array}$ & 0.174 & 0 & & 0.46 & 1 & \\
\hline 5 & 16.03 & 3 & & 8.326 & 0 & 3.638 & 0 & & 3.818 & 2 & (c) \\
\hline 6 & 177.6 & 2 & & 40.21 & 0 & 54.49 & 1 & & 20.80 & 1 & \\
\hline 7 & 1458 & 193 & & 152.1 & (c) & 617.9 & 6 & & 88.50 & 0 & \\
\hline 8 & $\geq 3000$ & 599 & (b) & 508.5 & 3 & 2799 & 885 & & 319.3 & 0 & \\
\hline 9 & $\geq 3000$ & 1389 & (b) & 1429 & 7 & $\geq 3000$ & 1178 & (b) & 935.6 & 2 & \\
\hline
\end{tabular}

(b) Has been run on a unique example

(c) Solution(s) is (are) missing due to infinite path(s) truncation

\begin{tabular}{|c|c|c|c|c|}
\hline \multicolumn{5}{|c|}{ Bertini with AMP arithmetic } \\
\hline$d$ & \begin{tabular}{|r} 
Proj \\
system $\mathcal{S}_{2}$ \\
$\mathrm{t}$
\end{tabular} & $\begin{array}{l}\text { ection } \\
\text { system } \mathcal{S}_{4} \\
\text { t }\end{array}$ & $\begin{array}{c}\text { Apparen } \\
\text { system } \mathcal{S}_{2} \\
\mathrm{t}\end{array}$ & $\begin{array}{l}\text { system } \mathcal{S}_{4} \\
\mathrm{t}\end{array}$ \\
\hline 4 & 2.332 & 1.804 (c) & 2.332 & 1.434 \\
\hline 5 & 147.8 & 13.888 & 147.852 & $15.01(\mathrm{c})$ \\
\hline 6 & $\geq 3000$ & 123.41 & 1005 & 165.7 \\
\hline 7 & $\geq 3000$ & 1089 (c) & $\geq 3000$ & 1147 \\
\hline 8 & $\geq 3000$ & $\geq 3000$ & $\geq 3000$ & $\geq 3000$ \\
\hline
\end{tabular}

Table 3. Isolating singularities of projection and apparent contour curves with subdivision. Input polynomials have degree $d$. The average running times $t$ are given in seconds together with the standard deviation $\sigma$.

\begin{tabular}{c|c|c||c|c|} 
& \multicolumn{2}{|c||}{ Projection } & \multicolumn{2}{c|}{ Apparent contour } \\
& system $\mathcal{S}_{2}$ & system $\mathcal{S}_{4}$ & system $\mathcal{S}_{2}$ & system $\mathcal{S}_{4}$ \\
$d$ & $\mathrm{t} \pm \sigma$ & $\mathrm{t} \pm \sigma$ & $\mathrm{t} \pm \sigma$ & $\mathrm{t} \pm \sigma$ \\
\hline 4 & $0.078 \pm 0.03$ & $0.759 \pm 0.02$ & $0.040 \pm 0.02$ & $1.509 \pm 1.97$ \\
5 & $0.351 \pm 0.13$ & $1.973 \pm 0.72$ & $0.251 \pm 0.23$ & $25.34 \pm 47.5$ \\
6 & $1.918 \pm 0.55$ & $6.442 \pm 3.07$ & $1.353 \pm 0.57$ & $11.38 \pm 6.98$ \\
7 & $9.528 \pm 3.92$ & $22.43 \pm 8.36$ & $124.1 \pm 142$ & $54.21 \pm 50.3$ \\
8 & $42.69 \pm 16.8$ & $57.00 \pm 16.4$ & $57.72 \pm 63.7$ & $99.22 \pm 89.3$ \\
9 & $163.3 \pm 111$ & $137.5 \pm 93$ & $54.74 \pm 33.3$ & $95.11 \pm 44.5$
\end{tabular}


the ball system can be solved with numerical methods. With homotopy it is more often complete and faster to solve the latter system than the sub-resultant system. A certified resolution is provided by a subdivision solver. In term of computational cost, such solvers are known to suffer from the increase of the dimension of the research space. However for high degrees of input polynomials, the price to pay for solving the sub-resultant system seems higher than the one induced by the increasing of number of variables.

Finally, our characterization could be extended to higher dimensions, for instance for singularities of the projection in $2 \mathrm{D}$ of a curve in $4 \mathrm{D}$ or the projection in $3 \mathrm{D}$ of a surface in $4 \mathrm{D}$.

\section{References}

1. Alberti, L., Mourrain, B., Técourt, J.P.: Isotopic triangulation of a real algebraic surface. J. Symb. Comput. 44(9), 1291-1310 (2009)

2. Arnold, V.I., Varchenko, A., Gusein-Zade, S.: Singularities of Differentiable Maps: Volume I: The Classification of Critical Points Caustics and Wave Fronts, vol. 82. Springer Science \& Business Media (1988)

3. Bank, B., Giusti, M., Heintz, J., Lecerf, G., Matera, G., Solernó, P.: Degeneracy loci and polynomial equation solving. Foundations of Computational Mathematics 15(1), 159-184 (2015), http://dx.doi.org/10.1007/s10208-014-9214-z

4. Bates, D.J., Hauenstein, J.D., Sommese, A.J., Wampler, II, C.W.: Adaptive multiprecision path tracking. SIAM J. Numer. Anal. 46(2), 722-746 (Feb 2008), http://dx.doi.org/10.1137/060658862

5. Berberich, E., Kerber, M., Sagraloff, M.: An efficient algorithm for the stratification and triangulation of an algebraic surface. Comput. Geom. Theory Appl. 43(3), 257-278 (2010)

6. Cheng, J., Lazard, S., Peñaranda, L., Pouget, M., Rouillier, F., Tsigaridas, E.: On the topology of real algebraic plane curves. Mathematics in Computer Science 4, 113-137 (2010)

7. Daouda, D.N., Mourrain, B., Ruatta, O.: On the computation of the topology of a non-reduced implicit space curve. In: ISSAC '08: Proceedings of the twenty-first international symposium on Symbolic and algebraic computation. pp. 47-54. ACM, New York, NY, USA (2008)

8. Dedieu, J.: Points fixes, zéros et la méthode de Newton. Mathématiques et Applications, Springer (2006)

9. Delanoue, N., Lagrange, S.: A numerical approach to compute the topology of the apparent contour of a smooth mapping from $R^{2}$ to $R^{2}$. Journal of Computational and Applied Mathematics 271, 267-284 (2014), http://www.sciencedirect.com/ science/article/pii/S0377042714001812

10. Demazure, M.: Bifurcations and catastrophes: geometry of solutions to nonlinear problems. Universitext, Springer, Berlin, New York (2000), École polytechnique.

11. El Kahoui, M.: Topology of real algebraic space curves. J. Symb. Comput. 43(4), 235-258 (2008)

12. Giusti, M., Lecerf, G., Salvy, B., Yakoubsohn, J.C.: On location and approximation of clusters of zeros: Case of embedding dimension one. Found. Comput. Math. 7(1), 1-58 (Feb 2007), http://dx.doi.org/10.1007/s10208-004-0159-5 
13. Hauenstein, J.D., Mourrain, B., Szanto, A.: Certifying isolated singular points and their multiplicity structure. In: Proceedings of the 2015 ACM on International Symposium on Symbolic and Algebraic Computation. pp. 213-220. ISSAC '15, ACM, New York, NY, USA (2015), http://doi.acm.org/10.1145/2755996.2756645

14. van der Hoeven, J.: Reliable homotopy continuation. Tech. rep., HAL (2011), http: //hal . archives-ouvertes.fr/hal-00589948/fr/

15. Imbach, R., Moroz, G., Pouget, M.: Numeric certified algorithm for the topology of resultant and discriminant curves. Research Report RR-8653, Inria (Apr 2015)

16. Kearfott, R., Xing, Z.: An interval step control for continuation methods. SIAM Journal on Numerical Analysis 31(3), 892-914 (1994)

17. Krantz, S.G., Parks, H.R.: A primer of real analytic functions, Basler Lehrbücher, vol. 4. Birkhäuser Verlag, Basel, Boston (1992)

18. Leykin, A., Verschelde, J., Zhao, A.: Newton's method with deflation for isolated singularities of polynomial systems. Theoretical Computer Science 359(13), 111122 (2006)

19. Mantzaflaris, A., Mourrain, B.: Deflation and certified isolation of singular zeros of polynomial systems. In: Proceedings of the 36th international symposium on Symbolic and algebraic computation. pp. 249-256. ISSAC '11, ACM, New York, NY, USA (2011), http://doi.acm.org/10.1145/1993886.1993925

20. Martin, B., Goldsztejn, A., Granvilliers, L., Jermann, C.: Certified parallelotope continuation for one-manifolds. SIAM Journal on Numerical Analysis 51(6), 3373$3401(2013)$

21. Moore, R.E., Kearfott, R.B., Cloud, M.J.: Introduction to Interval Analysis. Society for Industrial and Applied Mathematics, Philadelphia, PA, USA (2009)

22. Morgan, A.: Solving Polynominal Systems Using Continuation for Engineering and Scientific Problems. Society for Industrial and Applied Mathematics, Philadelphia, PA, USA (2009)

23. Mourrain, B., Pavone, J.: Subdivision methods for solving polynomial equations. Journal of Symbolic Computation 44(3), 292-306 (2009), http://www.sciencedirect.com/science/article/pii/s0747717108001168, ¡ce:title ¿Polynomial System Solving in honor of Daniel Lazardi/ce:title

24. Mourrain, B., Pion, S., Schmitt, S., Técourt, J.P., Tsigaridas, E.P., Wolpert, N.: Algebraic issues in Computational Geometry. In: Boissonnat, J.D., Teillaud, M. (eds.) Effective Computational Geometry for Curves and Surfaces, chap. 3, pp. 117-155. Mathematics and Visualization, Springer (2006)

25. Neumaier, A.: Interval methods for systems of equations. Cambridge University Press (1990)

26. Ojika, T., Watanabe, S., Mitsui, T.: Deflation algorithm for the multiple roots of a system of nonlinear equations. Journal of Mathematical Analysis and Applications 96(2), 463-479 (1983)

27. Plantinga, S., Vegter, G.: Isotopic approximation of implicit curves and surfaces. In: SGP '04: Eurographics/ACM SIGGRAPH Symposium on Geometry Processing. pp. 245-254 (2004)

28. Rouillier, F.: Solving zero-dimensional systems through the rational univariate representation. J. of Applicable Algebra in Engineering, Communication and Computing 9(5), 433-461 (1999)

29. Stahl, V.: Interval Methods for Bounding the Range of Polynomials and Solving Systems of Nonlinear Equations. Ph.D. thesis, Johannes Kepler University, Linz, Austria (1995)

30. Whitney, H.: On singularities of mappings of euclidean spaces. i. mappings of the plane into the plane. Annals of Mathematics 62(3), pp. 374-410 (1955) 